NASA Technical Memorandum 100171

\title{
A Computational Procedure for Automated Flutter Analysis
}

Durbha V. Murthy

University of Toledo

Toledo, Ohio

and

Krishna Rao V. Kaza

Lewis Research Center

Cleveland, Ohio

August 1987

\section{NMSA}




\title{
A COMPUTATIONAL PROCEDURE FOR AUTOMATED FLUTTER ANALYSIS
}

\author{
Durbha V. Murthy* \\ Department of Mechanical Engineering \\ University of Toledo \\ Toledo, Ohio 43606
}

and

Krishna Rao V. Kaza

National Aeronautics and Space Administration

Lewis Research Center

Cleveland, Ohio 44135

\section{SUMMARY}

A direct solution procedure for computing the flutter Mach number and the flutter frequency is applied to the aeroelastic analysis of propfans using a finite element structural model and an unsteady aerodynamic model based on a three-dimensional subsonic compressible lifting surface theory. An approximation to the Jacobian matrix that improves the efficiency of the iterative process is presented. The Jacobian matrix is indirectly approximated from approximate derivatives of the flutter matrix. Examples are used to illustrate the convergence properties. The direct solution procedure facilitates the automated flutter analysis in addition to contributing to the efficient use of computer time as well as the analyst's time.

\section{INTRODUCTION}

Flutter of propfans and other types of turbomachinery blading is an important phenomenon that has generated considerable interest. Flutter prevention has been a significant factor in the design of propfan blades. Flutter prevention is also significant for turbomachinery, particularly for unshrouded blades. As a result, a comprehensive flutter analysis procedure for rotating blade systems incorporating general mistuning and three dimensional subsonic aerodynamics is being developed at the NASA Lewis Research Center (refs. 1 to 3 ).

Research on the aeroelastic behavior of propfans has progressed from both the structural and the aerodynamic viewpoints. Because of the complex geometry of the propfan, structural modeling has shifted from an initial beam model (ref. 4) to advanced finite element models (refs. 2, 3, 5, and 6). Aerodynamic models evolved from two-dimensional lifting line theory (refs. 2 and 7 ) to three-dimensional lifting surface theory (ref. 8). Development of aerodynamic models with supersonic, transonic, and separated flow effects is also a continuing effort at present. Aeroelastic analysis of propfans (refs. 2 to 5 and 9) has kept pace with developments in structural and aerodynamic modeling. Comparisons with experimental data (refs. 2, 3, and 10) have resulted in reasonable confidence in analytical predictions.

*Resident Research Associate at NASA Lewis Research Center. 
With the recent advances in computer technology, automated design of propfan and turbomachinery blades using optimization techniques has become practical. Efforts in this direction have already begun (refs. 11 to 15). Design optimization employing flutter constraints requires repeated solution of the aeroelastic equations of motion to obtain the flutter parameters as the design is updated. For the optimization to be performed in a realistic period of time, an automated flutter analysis capability is essential. It is also desirable for the analysis to be computationally efficient in order to keep the central processing unit (CPU) time and the turnaround time within reasonable limits.

The conventional flutter analysis procedure, based on the $k$-method (ref. 16), casts the equations of motion as a complex linear eigenvalue problem where the real and imaginary parts of the eigenvalue are the effective damping and frequency respectively of the aeroelastic system. The solution consists of an inner-outer iteration loop to obtain the flutter Mach number and frequency. The inner iteration loop matches the assumed frequency to the calculated frequency while the outer loop iterates on the Mach number to obtain a zero effective damping.

A stumbling block in the development of a reliable automated procedure has been the need to track several eigenvalues through possible crossovers. Previous efforts in the development of automated flutter analysis procedures for fixed wings attempted to overcome this difficulty in two ways. One was to use eigenvalue derivative information to predict the crossovers and then keep track of them during the iteration (refs. 17 and 18). Apart from being cumbersome, this procedure required the derivatives of the aerodynamic force coefficients with respect to Mach number and assumed frequency. The other development was to use an accurate approximation to the eigenvalue which would retain the continuity of the eigenvalues over the desired range of Mach number and assumed frequency (ref. 19). This latter method is susceptible to the accuracy and the reliability of the approximation over a wide range and requires careful monitoring. In addition, the inner-outer iteration loop in the conventional procedure is computationally expensive. The inner loop involves the computation of the matrix of aerodynamic coefficients and the solution of a complex eigenvalue problem. This can degrade efficiency, particularly for problems involving structural and/or aerodynamic mistuning.

The present work explores an efficient and automated procedure which replaces the costly inner-outer double iteration loop with a single iteration loop. The need to track the eigenvalues is also avoided. This is accomplished by viewing the flutter problem as a complex implicit double eigenvalue problem and directly solving for the flutter Mach number and the flutter frequency by a quasi-Newton determinant iteration. The flutter eigenvalues are never calculated and the avoidance of eigenvalue tracking makes true automation possible. An accurate approximation to the Jacobian matrix which makes the iterative process very efficient is a new feature of the present procedure. The proposed procedure is tested by applying it to the flutter analys is of a propfan model at a number of rotational speeds.

While the idea of direct solution of the flutter equation is not new (refs. 16, 20, and 21), it has not been very popular. One reason may be that the alternative solution, involving flutter eigenvalues, has more physical appeal. However, the imperatives of automating the flutter analys is and reducing the computational expense of an inner-outer iteration loop make the direct 
solution more attractive. Some previous applications of direct solution of flutter equations by determinant iteration considered highiy simplified aerodynamic models. No results are avallable on the convergence and efficiency of the direct solution procedure considering more complex models such as kernel function aerodynamics, the computational expense of which makes such issues paramount. One of the objectives of this paper is to provide such results.

The present procedure also improves upon the previously published direct solution techniques of Hassig (ref. 16) and Cardani and Mantegazza (ref. 21). Hassig (ref. 16) uses a secant-based determinant iteration to obtain the matched flutter frequency and approximate subcritical rate of decay whereas the present procedure obtains both the matched flutter Mach number and the matched flutter frequency by a more efficient implementation of determinant iteration. Cardani and Mantegazza (ref. 21) solve the full set of nonlinear equations of motion without using determinant iteration to obtain the matched values of flutter Mach number, flutter frequency and the flutter mode simultaneously. In the procedure developed here, only two nonlinear equations are solved to obtain the flutter point and the flutter mode is obtained very efficiently with the information available from the final iteration. The Jacobian approximation and the direct solution procedure presented here are applicable to any aeroelastic stability analysis, including fixed wing, in a straightforward manner. The Jacobian approximation is also generally applicable to determinant iteration in any application.

NOMENCLATURE

[A] generalized aerodynamic force matrix

[B] system flutter matrix

D system flutter determinant

$D_{R} \quad$ real part of the flutter determinant

DI imaginary part of the fiutter determinant

$\left[J_{k}\right]$ Jacobian matrix

$k \quad$ counter used in determinant iteration

[Kg] generalized stiffness matrix

M Mach number

MF flutter Mach number

$\left[\mathrm{Mg}_{\mathrm{g}}\right]$ generalized mass matrix

$\Delta M_{k} \quad M_{F}(k-1)-M_{F}(k)$

\{qo\} flutter mode of vibration

\{q\} generalized coordinate vector 
t time

B0.75R blade setting angle at three-quarter radius

$\begin{array}{ll}\Delta \omega_{K} & \omega_{F}(k-1)-\omega_{F}(k) \\ \sigma_{r} & r \text {-th inter-group phase angle } \\ \Omega & \text { rotational speed of the propfan } \\ \omega & \text { frequency of vibration } \\ \omega F & \text { flutter frequency }\end{array}$

FORMULATION OF THE FLUTTER ANALYSIS PROBLEM

The computational procedure to be presented is applied to the analytical formulation described in detail in references 2 and 3 . This formulation is applicable to the flutter analysis of a single rotor propfan containing an arbitrary number of blades rotating at a fixed speed in an axial flow. The structure is modeled by finite elements. The aerodynamic model (ref. 8 ) is based on a three-dimensional subsonic compressible lifting surface theory.

For simplicity, the effect of steady deformations due to aerodynamic loads on the flutter boundary is neglected. The error introduced by ignoring the steady aerodynamic deformations is studied in reference 2 and is shown to be small enough to produce an approximate flutter point. In particular, the effect of the steady aerodynamic deformations on the flutter Mach number is not very significant. Thus, the approximate flutter analysis neglecting steady aerodynamic deformations is suitable for use in design optimization procedures requiring repeated efficient execution of the flutter analysis. The optimal design can be easily checked for the flutter condition by using the refined flutter analysis with steady deformations and the conventional procedure.

The propfan is assumed to have identical groups of blades symmetrically distributed about a rigid disk. The linearized aeroelastic equations of motion are then uncoupled for different inter-group phase angle modes. The flutter Mach number for the propfan is then the lowest Mach number at which one of the intergroup phase angle modes becomes unstable. For a given intergroup phase angle $\sigma_{r}$, the equations of motion, after modal transformation, can be written in the form (ref. 3 ):

$$
\left[M_{g}\right]\{\ddot{q}\}+\left[K_{g}\right]\{q\}=[A(M, \omega)]\{q\}
$$

where $\left[M_{g}\right]$ is the generalized mass matrix, $\left[K_{g}\right]$ is the generalized stiffness matrix, $\{q\}$ the generalized coordinate vector and $[A(M, \omega)]$ the generalized aerodynamic matrix for a group of blades. The aerodynamic matrix $[A(M, \omega)]$ is usually valid only for simple harmonic motion of the airfoil. For full details of the construction of these quantities, the reader is referred to references 2 and 3 . 
For simple harmonic motion,

$$
\{q\}=\left\{q_{0}\right\} e^{i \omega t}
$$

Equation (1) now leads to

$$
\left(-\omega^{2}\left[M_{g}\right]+\left[K_{g}\right]-[A(M, \omega)]\right)\left\{q_{0}\right\}=\{0\}
$$

The conventional procedure for obtaining the flutter Mach number and frequency, outlined in references 2 and 3 and illustrated in figure 1 (reproduced from ref. 2 ignoring the computation of steady deformations due to aerodynamic loads), is as follows. The aerodynamic matrix is evaluated at an assumed Mach number and an assumed frequency and then equation ( 3 ) is solved for all the eigenvalues, $\omega^{2}$. This procedure has been implemented for propfans in a program called ASTROP3. In general, these eigenvalues are complex. The real and imaginary parts of $i \omega$ represent the effective damping and frequency, respectively. The assumed frequency is varied until it is equal to the frequency corresponding to the eigenvalue with the least effective damping. This frequency matching forms the inner iteration. When this iteration reaches convergence, the Mach number is varied until the effective damping of the eigenvalue corresponding to the matched frequency is equal to zero. This forms the outer iteration. The flutter Mach number and the flutter frequency are obtained at the convergence of the outer iteration.

The conventional procedure cannot be reliably automated because it requires the preservation of the identity or ordering of the eigenvalues over a wide range of assumed frequencies and Mach numbers. Most eigensolution routines do not compute the eigenvalues in any particular order and the sorting of eigenvalues by frequency or magnitude does not usually preserve the continuity. Loss of continuity necessitates user intervention and complicates the automated analysis.

In order to alleviate the se problems, a direct solution of equation (3) viewed as an implicit double eigenvalue problem is proposed and described below.

\section{DIRECT SOLUTION OF THE FLUTTER EQUATION}

When the dependence of the aerodynamic matrix on the assumed Mach number and frequency is considered explicitly, equation (3) can be viewed as an implicit double eigenvalue problem. If $M_{F}$ is the flutter Mach number and $\omega F$ the flutter frequency, then equation (3) leads to

$$
[B]\left\{q_{0}\right\}=\{0\}
$$

where $[B]=-\omega F^{2}\left[M_{g}\right]+\left[K_{g}\right]-\left[A\left(M_{F}, \omega_{F}\right)\right]$

In general, the aerodynamic matrix $\left[A\left(M_{F}, \omega_{F}\right)\right]$ is a transcendental function of the assumed frequency and Mach number. $M_{F}$ and $\omega_{F}$ are the two eigenvalues of equation (4). Only real values of $M_{F}$ and $\omega_{F}$ are of interest. The two eigenvalues $M_{F}$ and $\omega_{F}$ are coupled since the aerodynamic matrix is 
complex. Cardani and Mantegazza (ref. 21) proposed a direct solution of equation (4) and a normalizing condition, treating $M_{F}$, wF and the components of \{q0\} as unknowns. For a nontrivial flutter mode, we have the characteristic equation

$$
\operatorname{det}\left(-\omega_{F}^{2}\left[M_{g}\right]+\left[K_{g}\right]-\left[A\left(M_{F}, \omega_{F}\right)\right]\right)=0
$$

Let

$$
\begin{aligned}
D & =\operatorname{det}\left(-\omega_{F}^{2}\left[M_{g}\right]+\left[K_{g}\right]-\left[A\left(M_{F}, \omega_{F}\right)\right]\right) \\
& =D_{R}\left(M_{F}, \omega_{F}\right)+i D_{I}\left(M_{F}, \omega_{F}\right)
\end{aligned}
$$

where $D_{R}$ and $D_{I}$ are the real and the imaginary parts of the characteristic determinant $D$ respectively. Then, at flutter condition,

$$
\begin{aligned}
& D_{R}\left(M_{F}, \omega_{F}\right)=0 \\
& D_{I}\left(M_{F}, \omega_{F}\right)=0
\end{aligned}
$$

Equations (7) consist of two equations in two unknowns, $M_{F}$ and $\omega_{F}$. These equations can be solved by any of the methods for solving a system of nonlinear equations. When equations (7) are solved for $M_{F}$ and $\omega_{F}$, equation (4) is satisfied and no further iterations are required for the purpose of matching assumed and computed quantities. This procedure is illustrated in figure 2. Once $M_{F}$ and $\omega_{F}$ are found, inverse iteration can be used to find the flutter mode.

In contrast to the conventional procedure, the direct solution outlined here eliminates the need to track eigenvalues to determine the flutter point. In addition to this important benefit, the double iteration on the complex eigensolution is replaced by a single solution of a system of two real nonlinear equations. The Mach number and frequency are varied simultaneously in this procedure rather than one at a time as in the conventional procedure. Thus the flutter Mach number and the flutter frequency are determined simultaneously.

The formulation of a transcendental double eigenvalue problem in preference to a linear single eigenvalue problem may seem to defeat the objective of increased efficiency, even if it is more suitable for automation. However, the price to be paid is not as great as it may seem because the transcendental eigenvalue problem needs to be solved for only one set of eigenvalues in most cases, whereas the linear eigenvalue problem has to be repeatedly solved for all the eigenvalues which equal in number to the number of assumed mode shapes.

\section{NUMERICAL SCHEME FOR DIRECT SOLUTION}

Equations (7) may be solved for $M_{F}$ and $\omega_{F}$ by Newton's method. The iterative scheme for Newton's method is

$$
\left\{\begin{array}{l}
M_{F} \\
\omega_{F}
\end{array}\right\}_{(k+1)}=\left\{\begin{array}{l}
M_{F} \\
\omega_{F}
\end{array}\right\}_{(k)}-\left[J_{k}\right]^{-1}\left\{\begin{array}{l}
D_{R} \\
D_{I}
\end{array}\right\}_{(k)}
$$


where $k$ is the iteration number and $\left[J_{k}\right]$ is the Jacobian matrix and is given by

$$
\left[J_{k}\right]=\left[\begin{array}{ll}
D_{k R, M_{F}} & D_{k R, \omega_{F}} \\
D_{k I, M_{F}} & D_{K I, \omega_{F}}
\end{array}\right]
$$

The Jacobian $\left[J_{k}\right]$ is expensive to compute because the evaluation of the aerodynamic matrix [ $\left.\hat{A}_{k}\right]$ is computationally intensive. Several quasi-Newton algorithms which approximate the Jacobian in various ways are available. In the following, a quasi-Newton algorithm is proposed that is more efficient than the currently available algorithms for the determination of the flutter Mach number and the flutter frequency. The numerical scheme is based on the hypothesis that approximating the derivatives of the flutter matrix [ $\left.B_{k}\right]$ provides a more accurate approximation to the Jacobian matrix $\left[J_{k}\right]$ than directly approximating the derivatives of the characteristic determinant. The numerical scheme based on this hypothesis approaches the Newton's method in its superior convergence characteristics with the same cost per iteration as the secant method.

The iterative scheme of equation ( 8 ) is first transformed in order to explicitly determine the relation between the derivatives of the characteristic determinant and those of the flutter matrix. This is accomplished by employing the following remarkable result, known as the Trace theorem, proved by Lancaster (ref. 22): If the elements of $[B(\tilde{a})]$ are differentiable functions of $\tilde{a}$, then for any $\tilde{a}$ for which the characteristic determinant $D(\tilde{a}) \neq 0$,

$$
D, \tilde{a}=D \cdot \operatorname{trace} \text { of }\left([B]^{-1}[B], \tilde{a}\right)
$$

When $\tilde{a}$ is successively set to $M_{F}$ and $\omega_{F}$, equation (10) leads to

$$
\begin{aligned}
& D_{k, M_{F}}=D_{K R, M_{F}}+i D_{k I, M_{F}}=D_{k} \cdot \text { trace of }\left(\left[B_{k}\right]^{-1}\left[B_{k}\right], M_{F}\right) \\
& D_{k, \omega F}=D_{k R, \omega F}+i D_{k I, \omega F}=D_{k} \cdot \text { trace of }\left(\left[B_{k}\right]^{-1}\left[B_{K}\right], \omega_{F}\right)
\end{aligned}
$$

The iterative scheme of equation ( 8 ) can then be written as

$$
\left\{\begin{array}{l}
M_{F} \\
\omega_{F}
\end{array}\right\}_{(k+1)}=\left\{\begin{array}{l}
M_{F} \\
\omega_{F}
\end{array}\right\}_{(k)}-\frac{1}{\left({ }_{R, M_{F}{ }_{I}, \omega_{F}}-g_{\left.R, \omega_{F}{ }_{I}, M_{F}\right)_{(k)}}\right.}\left\{\begin{array}{c}
g_{I}, \omega_{F} \\
-g_{I, M_{F}}
\end{array}\right\}_{(k)}
$$

where

$$
\begin{aligned}
& \left(g, M_{F}\right)_{k}=\operatorname{trace} \text { of }\left\langle\left[B_{k}\right]^{-1}\left[B_{k}\right], M_{F}\right)=\left(g R, M_{F}+i g I, M_{F}\right)_{k} \\
& \left(g, \omega_{F}\right)_{k}=\text { trace of }\left(\left[B_{k}\right]^{-1}\left[B_{k}\right], \omega_{F}\right)=\left(g_{R, \omega_{F}}+i g_{I}, \omega_{F}\right)_{k}
\end{aligned}
$$


The iterative formula of equation (12) does not require the determinant of the coefficient matrix $\left[B_{k}\right]$ of the flutter equation (4). However, $\left[B_{k}\right]^{-1}$ is required to compute $g, M_{F}$ and $g, \omega_{F}$. The computational cost for the inversion of $\left[B_{k}\right]$ is usually negligible in comparison to that of forming the matrix $\left[B_{k}\right]$.

The derivatives $\left[B_{k}\right], M_{F}$ and $\left[B_{k}\right], \omega F$ are approximated by following a reasoning similar to that employed in deriving Broyden's method (ref. 23).

Let $\Delta M_{k}=M_{F}(k-1)-M_{F}(k)$ and $\Delta \omega_{k}=\omega_{F}(k-1)-\omega_{F}(k)$. The derivatives are approximated in the direction of the last move to satisfy

$$
\left[B_{k-1}\right]=\left[B_{k}\right]+\left[B_{k}\right], M_{F} \cdot \Delta M_{k}+\left[B_{k}\right], \omega_{F} \cdot \Delta \omega_{k}
$$

and are assumed to be unchanged in the direction orthogonal to the last move and this implies

$$
\begin{array}{r}
{\left[B_{k}\right], M_{F}\left(M_{F}-M_{F}(k)\right)+\left[B_{k}\right], \omega_{F}\left(\omega_{F}-\omega_{F}(k)\right)=\left[B_{k-1}\right], M_{F}\left(M_{F}-M_{F}(k)\right)} \\
+\left[B_{k-1}\right], \omega_{F}\left(\omega_{F}-\omega_{F}(k)\right)
\end{array}
$$

for any $M_{F}$ and $\omega_{F}$ satisfying:

$$
\left(M_{F}-M_{F}(k)\right) \cdot \Delta M_{k}+\left(\omega_{F}-\omega_{F}(k)\right) \cdot \Delta \omega_{k}=0
$$

Equations (14) and (15) are solved to obtain

$$
\begin{aligned}
& {\left[B_{k}\right], M_{F}=\frac{\left(\left[B_{k-1}\right]-\left[B_{k}\right]\right) \Delta M_{k}+\left(\left[B_{k-1}\right], M_{F} \cdot \Delta \omega_{k}-\left[B_{k-1}\right], \omega_{F} \cdot \Delta M_{k}\right) \Delta \omega_{k}}{\Delta M_{k}^{2}+\Delta \omega_{k}^{2}}} \\
& {\left[B_{k}\right]_{, \omega_{F}}=\frac{\left(\left[B_{k-1}\right]-\left[B_{k}\right]\right) \Delta \omega_{k}-\left(\left[B_{k-1}\right], M_{F} \cdot \Delta \omega_{k}-\left[B_{k-1}\right] \omega_{F} \cdot \Delta M_{k}\right) \Delta M_{k}}{\Delta M_{k}^{2}+\Delta \omega_{k}^{2}}}
\end{aligned}
$$

It was expected that equations (13), in conjunction with equations (16) and (17), approximate the Jacobian matrix accurately enough to obviate the need to update the Jacobian in the entire range of convergence. Results in the next section show that this is indeed the case for the examples studied, even when large moves were made during the iteration. is:

The proposed numerical scheme for direct solution of the flutter equation

(1) Obtain initial estimates $M_{F}(0)$ and $\omega_{F}(0)$ for the flutter Mach number and flutter frequency.

(2) Obtain initial estimates for the derivatives $\left[B_{0}\right], M_{F}$ and $\left[B_{0}\right], \omega_{F}$. These are best obtained by computing finite difference approximations.

(3) Compute new estimates $M_{F(k+1)}$ and $\omega_{F}(k+1), k=0,1,2, \ldots$ using equation (12). 
(4) Test for convergence and stop if converged.

(5) Increment $k=k+1$.

(6) Compute updated derivatives $\left[B_{k}\right], M_{F}$ and $\left[B_{k}\right], \omega_{F}$ using equations (16) and (17).

(7) Repeat from step 3.

$\left[B_{k}\right]^{-1}$ from the final iteration of the above procedure can be used in an inverse iteration scheme to determine the flutter mode at little additional cost. Thus, if $\left\{q_{0 F}\right\}$ is the required flutter mode and $\{v\}$ is any vector that is not totally deficient in $\left\{q_{0 F}\right\}$, then the following inverse iteration scheme is used to obtain the flutter mode:

$$
\begin{gathered}
\left\{q_{O F}\right\}_{1}=[B]^{-1}\{v\} \\
\left\{q_{O F}\right\}_{m+1}=[B]^{-1}\left\{q_{O F}\right\}_{m}, \quad m=1,2, \ldots
\end{gathered}
$$

Normally, one step of the inverse iteration scheme is sufficient to obtain flutter mode within a reasonable accuracy.

\section{RESULTS AND DISCUSSION}

To demonstrate the current procedure, flutter boundary calculations were performed for two configurations of propfan rotors described in references 2 and 3. The first configuration consists of eight identical blades, designated SR3C-X2, made of graphite-ply/epoxy-matrix material. The second configuration is an alternately mistuned rotor with eight blades. The rotor was constructed by alternately mounting two sets of composite blades. The first set of four blades, SR3C-X2, are the same as those on the rotor of the first configuration. The second set of blades, SR3C-3, are identical to the first set except for the ply angles of the composite material. For details on the blade models, the reader is referred to references 2 and 3 . In this report, the first configuration is called the tuned rotor and the second configuration the mistuned rotor.

To validate the current procedure and the computer program, flutter boundaries were calculated at various rotational speeds for both the tuned and mistuned rotors. Convergence was considered to have been obtained when the solution did not change to four significant digits in successive iterations. During the iteration process, the value of the Mach number sometimes turned out to be supersonic at the tip. No aerodynamic analysis can be performed at such Mach numbers using the current aerodynamic model. When such intermediate solutions (including those involving negative Mach numbers) arose, the move length was cut in steps of 80 percent until an acceptable Mach number was obtained. This technique was found to be effective in driving the solution to the desired range without an excessive penalty in the number of iterations. The results obtained with the present procedure starting with various initial guesses are compared with the "exact" flutter boundaries obtained using the conventional procedure, at two rotational speeds each for tuned and mistuned rotors. The flutter boundaries are in excellent agreement for these cases, thus validating the accuracy of the procedure and the convergence criteria. 
The typical progress of iteration, for the initial guesses for flutter Mach number and flutter frequency of 0.5 and $310 \mathrm{~Hz}$ respectively, with the present direct solution procedure and the conventional procedure, is shown in table I. Note that the conventional procedure relies on user interaction and judgment. The progress of iteration shown for the conventional procedure is typical. The current procedure, in addition to being suitable for automation, is also more efficient as evidenced by the considerably smaller number of analysis steps. Thus both the CPU time and analyst man-hours are considerably reduced using the present procedure.

To judge the accuracy of the approximate Jacobian, the nonlinear equations (7) were solved by Newton's method, the present numerical procedure and by alternate quasi-Newton methods such as the multi-point secant method (ref. 24) (implemented in IMSL routine ZSCNT), modified Powell's algorithm (implemented in IMSL routine ZSPOW) and by Broyden's method (ref. 23). For the cases tested, the present procedure outperformed all three alternate methods in terms of efficiency. Figures 3,4 , and 5 illustrate the convergence behavior of the current procedure in comparison to Newton's method and the multipoint secant method. For the cases shown, the "exact" flutter Mach number was 0.641 and the "exact" flutter frequency was $294 \mathrm{~Hz}$. Even though the characteristic determinant $D_{k}$ is never calculated in the current procedure, the variation of the absolute value of $D_{k}$ with each iteration is shown in figure 3 for comparison with the Newton's method and the secant method. The initial values for $M_{F}$ and $\omega_{F}$ were 0.65 and $330 \mathrm{~Hz}$ respectively. The determinant value has been scaled so that $1.0 \leq\left|D_{0}\right| \leq 10.0$. It is seen that the iteration history using the current numerical procedure closely resembles that of the Newton's method indicating the accuracy of the approximation proposed here for the Jacobian matrix. In contrast, the secant approximation for the Jacobian matrix almost doubles the number of iterations required for convergence in this case.

The range of convergence is an important factor in any iterative procedure since it has an important effect on how closely the initial solution must approximate the final solution. Figure 4 shows the number of flutter matrix evaluations required for convergence for various initial guesses for $M_{F}$ and a fixed initial guess for $\omega_{F}=310 \mathrm{~Hz}$. The range of Mach number convergence is seen to be from $M_{F}=0.2$ to $M_{F}=0.8$. Figure 5 similarly shows the number of flutter matrix evaluations required for convergence for a fixed initial guess for $M_{F}$ of 0.65 and various initial guesses for $\omega F$. The frequency range of convergence with the current procedure, $\omega_{F}=230 \mathrm{~Hz}$ to $\omega_{F}=350 \mathrm{~Hz}$, is seen to be slightly larger than that with the secant method. From these figures, it can be stated that the present procedure has a large radius of convergence.

The results obtained show that a fair initial guess would converge to the "exact" flutter point at the expense of about 5 to 10 flutter matrix evaluations. Table II shows the CPU times on the CRAY-XMP that were required to obtain the flutter boundary for the case of a good initial guess and a poor initial guess. The CPU times for one flutter eigenvalue analysis at a given set of Mach number and assumed frequency are al so shown for comparison. With a good initial guess, the flutter Mach number and the flutter frequency can be obtained for two or three times the cost of a single eigenanalysis. The present procedure is much less expensive in terms of CPU time as well as analyst's man-hours than the conventional procedure, even though precise comparisons have not been made. However, the primary motivation for the current 
procedure has been the feasibility of automation rather than the computational expense.

The current procedure may not find the lowest flutter Mach number, if more than one structural modes were to flutter in the Mach number and frequency range of interest for the selected intergroup phase angle mode. Under these circumstances, one will be forced to search the entire range of interest for the roots of the equations (7) starting with different initial guesses and this is not amenable to an efficient automated procedure. However, it is expected that such is rarely the case for tuned or alternately mistuned propfans. This is not a major limitation for two other reasons: (1) the frequency interval in which flutter speeds occur is usually determined early in the design phase and (2) the search domain can be considerably reduced after a few orientational runs.

\section{CONCLUSIONS}

A direct solution of the equations of motion is demonstrated to be a reliable automated flutter analysis procedure if steady aerodynamic deformations are ignored. The direct solution method replaces the inner-outer iteration loop of the conventional procedure by a single iteration loop. A numerical procedure, based on an accurate and efficient approximation to the Jacobian matrix, is presented. The procedure is straight-forward in concept and results for test cases show good convergence properties. Since the procedure is iterative, it is particularly suitable for design optimization. This is because, as the optimal design is evolved, the flutter solution is expected to change incrementally from design to design, so that the previous solution provides good estimates for the current solution.

\section{REFERENCES}

1. Kaza, K.R.V.; and Kielb, R.E.: Flutter of Turbofan Rotors with Mistuned Blades. AIAA J., vol. 22, no. 11, Nov. 1984, pp. 1618-1625.

2. Kaza, K.R.V., et al.: Analytical Flutter Investigation of a Composite Propfan Mode1. 28th Structures, Structural Dynamics and Materials Conference, Part 2A, AIAA, 1987, pp. 84-97. (NASA TM-88944).

3. Kaza, K.R.V., et al.: Analytical and Experimental Investigation of Mistuning on Propfan Flutter. 28th Structures, Structural Dynamics and Materials Conference, Part 2A, AIAA, 1987, pp. 98-110. (NASA TM-88959).

4. Mehmed, 0., et al.: Bending-Torsion Flutter of a Highly Swept Advanced Turboprop. NASA TM-82975, 1981.

5. Hirschbein, M., et al.: Structural and Aeroelastic Analysis of the SR-7L Propfan. NASA TM-86877, 1985.

6. Kosmatka, J.B.; and Friedmann, P.P.: Structural Dynamic Modeling of Advanced Composite Propellers by the Finite Element Method. 28th Structures, Structural Dynamics and Materials Conference, AIAA, 1987, pp. $111-124$. 
7. Turnberg, J.E.: Classical Flutter Stability of Swept Propellers. AIAA Paper 83-0847, May 1983.

8. Williams, M.H.; and Hwang, C.C.: Three Dimensional Unsteady Aerodynamics and Aeroelastic Response of Advanced Turboprops. 27th Structures, Structural Dynamics and Materials Conference, Part 2, AIAA, 1986, pp. $116-124$.

9. Ramsey, J.K.; and Kaza, K.R.V.: Concentrated Mass Effects on the Flutter of a Composite Advanced Turboprop Mode1. NASA TM-88854, 1986.

10. Mehmed, O.; and Kaza, K.R.V.: Experimental Classical Flutter Results of a Composite Advanced Turboprop Mode1. NASA TM-88792, 1986.

11. Crawley, E.F.; and Hall, K.C.: Optimization and Mechanisms of Mistuning in Cascades. J. Eng. Gas Turbines Power, vol. 107, no. 2, Apr. 1985, pp. $418-426$.

12. Nissim, E.; and Haftka, R.T.: Optimization of Cascade Blade Mistuning, Part II: Global Optimum and Numerical Optimization. AIAA J., vol. 23, no. 9, Sept. 1985, pp. 1402-1410.

13. Murthy, D.V.; and Haftka, R.T.: Optimization of Cascade Blade Mistuning Under Flutter and Forced Response Constraints. Recent Experiences in Multidisciplinary Analysis and Optimization, J. Sobieski, ed., NASA CP-2327-PT-1, 1984.

14. Brown, K.W.; Hirschbein, M.S.; and Chamis, C.C.: Finite Element Engine Blade Structural Optimization. 26th Structures, Structural Dynamics and Materials Conference, Part 1, AIAA, 1985, pp. 793-803.

15. Brown, K.; Harvey, P.R.; and Chamis, C.C.: Structural Tailoring of Advanced Turboprops. 28th Structures, Structural Dynamics and Materials Conference, Part 1, AIAA, 1987, pp. 827-837.

16. Hassig, H.J.: An Approximate True Damping Solution of the Flutter Equation by Determinant Iteration. J. Aircr., vol. 8, no. 11, Nov. 1971, pp. 885-889.

17. Rudisill, C.S.; and Cooper, J.L.: An Automated Procedure for Determining the Flutter Velocity. J. Aircr., vol. 10, no. 7, July 1973, pp. 442-444.

18. Bhatia, K.G.: An Automated Method for Determining the Flutter Velocity and the Matched Point. J. Aircr., vol. 11, no. 1, Jan. 1974, pp. 21-27.

19. Desmarais, R.N.; and Bennett, R.M.: An Automated Procedure for Computing Flutter Eigenvalues. J. Aircr., vol. 11, no. 2, Feb. 1974, pp. 75-80.

20. Scanlan, R.H.; and Rosenbaum, R.: Introduction to the Study of Aircraft Vibration and Flutter. Dover Publications, New York, 1968.

21. Cardani, C.; and Mantegazza, P.: Continuation and Direct Solution of the Flutter Equation. Comput. Struct., vol. 8, no. 2, Apr. 1978, pp. 185-192. 
22. Lancaster, P.: Algorithms for Lambda-Matrices. Numerische Mathematik, vol. 6, 1964, pp. 388-394.

23. Johnston, R.L.: Numerical Methods. Wiley, 1982.

24. Wolfe, P.: The Secant Method for Simultaneous Nonl inear Equations. Communications of the ACM, vol. 2, no. 12, DeC. 1969, pp. 12-13.

TABLE I. - PROGRESS OF ITERATION

$\left[\Omega=5280 \mathrm{rpm}, \beta_{0.75 R}=61.6^{\circ}, \sigma_{r}=225^{\circ}.\right]$

\begin{tabular}{|c|c|c|c|c|}
\hline \multirow[t]{2}{*}{ Count } & \multicolumn{2}{|c|}{ Conventional procedure } & \multicolumn{2}{|c|}{ Present procedure } \\
\hline & $M$ & $\begin{array}{l}\omega, \\
H_{2}\end{array}$ & M & $\begin{array}{l}\omega, \\
H z\end{array}$ \\
\hline $\begin{array}{c}1 \\
2 \\
3 \\
4 \\
5 \\
6 \\
7 \\
8 \\
9 \\
10 \\
11 \\
12 \\
13 \\
14 \\
15 \\
16 \\
17 \\
18\end{array}$ & $\begin{array}{r}0.500 \\
.500 \\
.500 \\
.500 \\
.700 \\
.700 \\
.700 \\
.700 \\
.616 \\
.616 \\
.616 \\
.616 \\
.640 \\
.640 \\
.640 \\
.641 \\
.641 \\
0.641\end{array}$ & $\begin{array}{l}310.0 \\
267.5 \\
268.9 \\
268.9 \\
268.9 \\
299.8 \\
298.9 \\
298.9 \\
286.3 \\
290.1 \\
290.5 \\
290.4 \\
292.9 \\
293.9 \\
294.0 \\
293.9 \\
294.1 \\
294.1\end{array}$ & $\begin{array}{r}0.500 \\
.499 \\
.500 \\
.701 \\
.590 \\
.641 \\
.642 \\
.641 \\
a .641\end{array}$ & $\begin{array}{l}310.0 \\
310.0 \\
313.0 \\
289.7 \\
287.7 \\
293.6 \\
294.1 \\
294.1 \\
294.1\end{array}$ \\
\hline
\end{tabular}

${ }^{\text {aConverged. }}$

TABLE II. - CPU SECONDS FOR AUTOMATED FLUTTER ANALYSIS

\begin{tabular}{|c|c|c|c|}
\hline & \multirow{2}{*}{$\begin{array}{l}\text { Eigensolution } \\
\text { at a single } \\
\text { set of } \\
M \text { and } w\end{array}$} & \multicolumn{2}{|c|}{$\begin{array}{c}\text { Direct solution to find } \\
M_{F} \text { and } \omega_{F}\end{array}$} \\
\hline & & $\begin{array}{l}\text { Good } \\
\text { initial } \\
\text { guessa }\end{array}$ & $\begin{array}{l}\text { Poor } \\
\text { initial } \\
\text { guessa }\end{array}$ \\
\hline $\begin{array}{l}\text { Tuned rotor }{ }^{b}-8 \text { blades; } \\
6 \text { modes } / \mathrm{bl} \text { lade; } 5280 \mathrm{rpm} ; \\
\sigma_{r}=225^{\circ}\end{array}$ & 4.332 & $\begin{array}{c}10.356 \\
(M=0.70 \\
\omega=310 \mathrm{~Hz})\end{array}$ & $\begin{array}{c}22.146 \\
(M=0.45 \\
\omega=340 \mathrm{~Hz})\end{array}$ \\
\hline $\begin{array}{l}\text { Mistuned rotorc }-8 \text { blades; } \\
4 \text { groups; } 2 \text { modes } / \text { blade; } \\
5190 \mathrm{rpm} ; \sigma_{r}=90^{\circ}\end{array}$ & 10.020 & $\begin{array}{c}22.084 \\
\langle M=0.65 \\
\omega \quad 310 \mathrm{~Hz})\end{array}$ & $\begin{array}{c}31.970 \\
(M=0.5 \\
\omega=340 \mathrm{~Hz})\end{array}$ \\
\hline
\end{tabular}

Initial guesses are given in parentheses.

b"Exact" $M_{F}=0.641$ and "exact" $\omega_{F}=294 \mathrm{~Hz}$.

c"Exact" $M_{F}=0.718$ and "exact" $\omega_{F}=285 \mathrm{~Hz}$. 


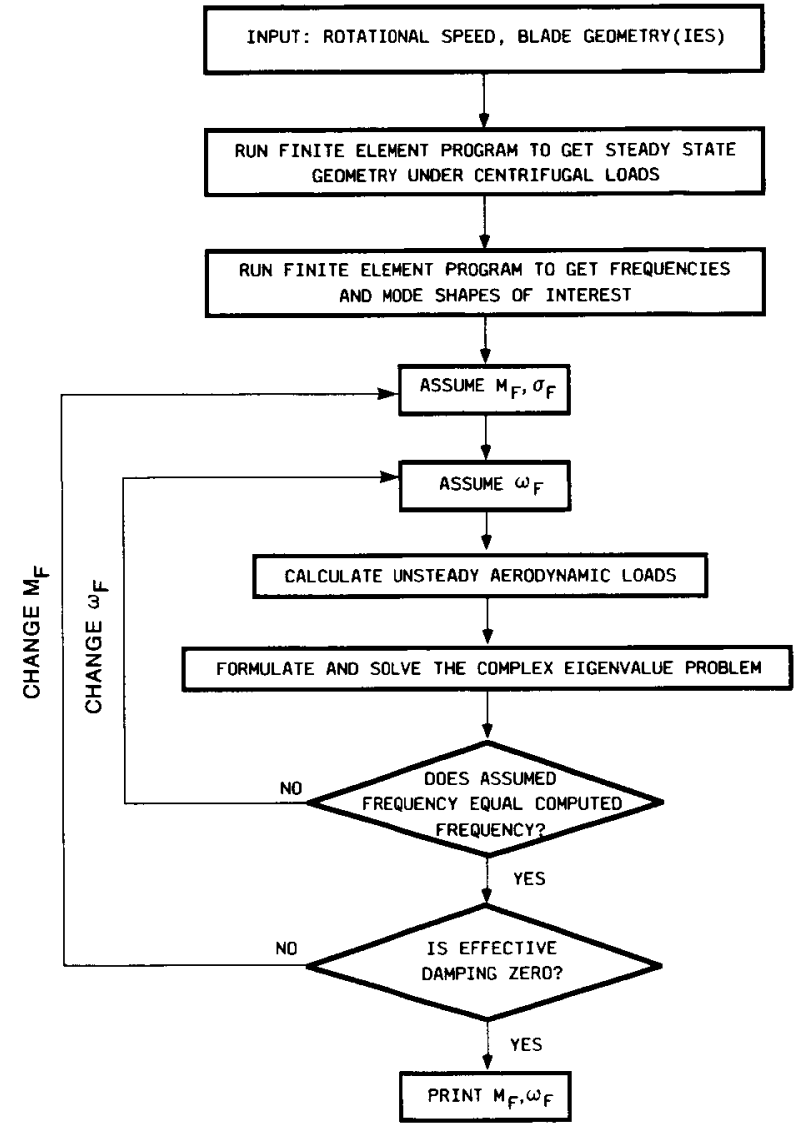

FIGURE 1. - CONVENTIONAL PROPFAN FLUTTER ANALYSIS.

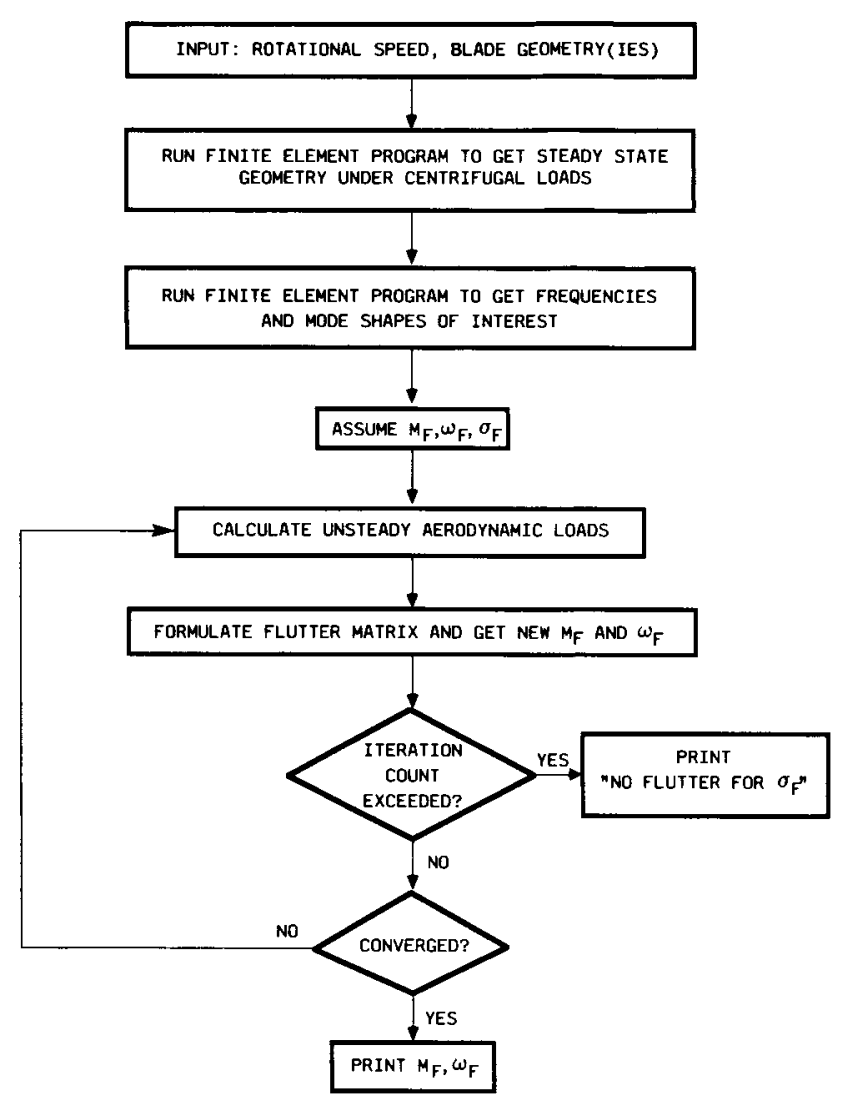

FIGURE 2. - AUTOMATED PROPFAN FLUTTER ANALYSIS. 

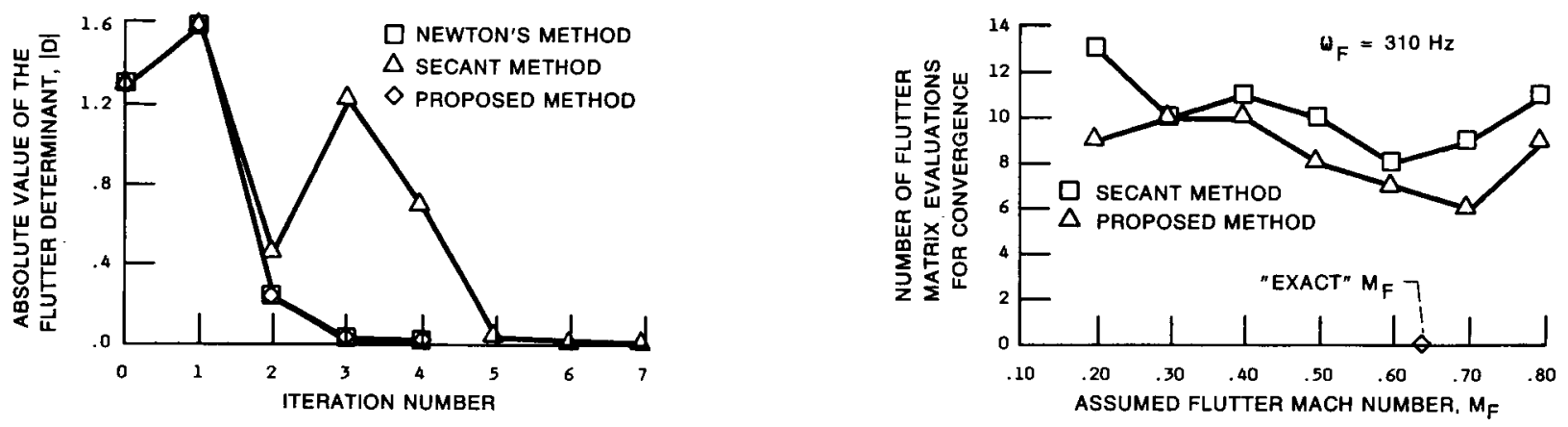

FIGURE 3. - VARIATION OF THE ABSOLUTE VALUE OF THE DETERMINANT WITH ITERATION NUMBER

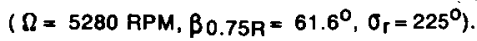

FIGURE 4. - CONVERGENCE OF DIRECT SOLUTION FOR VARIOUS INITIAL GUESSES AT FLUTTER MACH NUMBER.

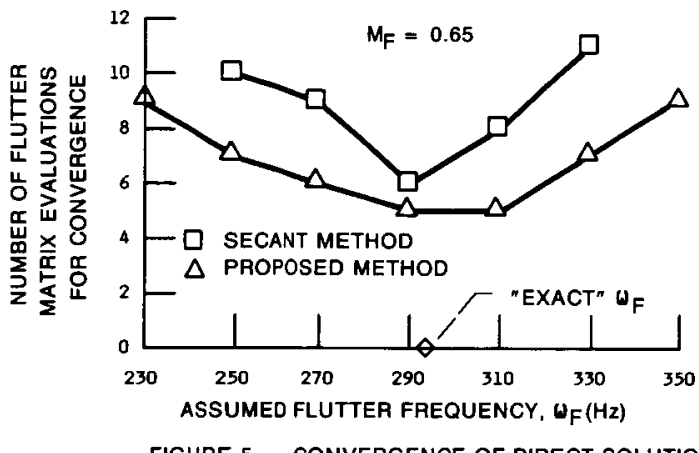

FIGURE 5. - CONVERGENCE OF DIRECT SOLUTION

FOR VARIOUS INITIAL GUESSES AT FLUTTER

FREQUENCY. 


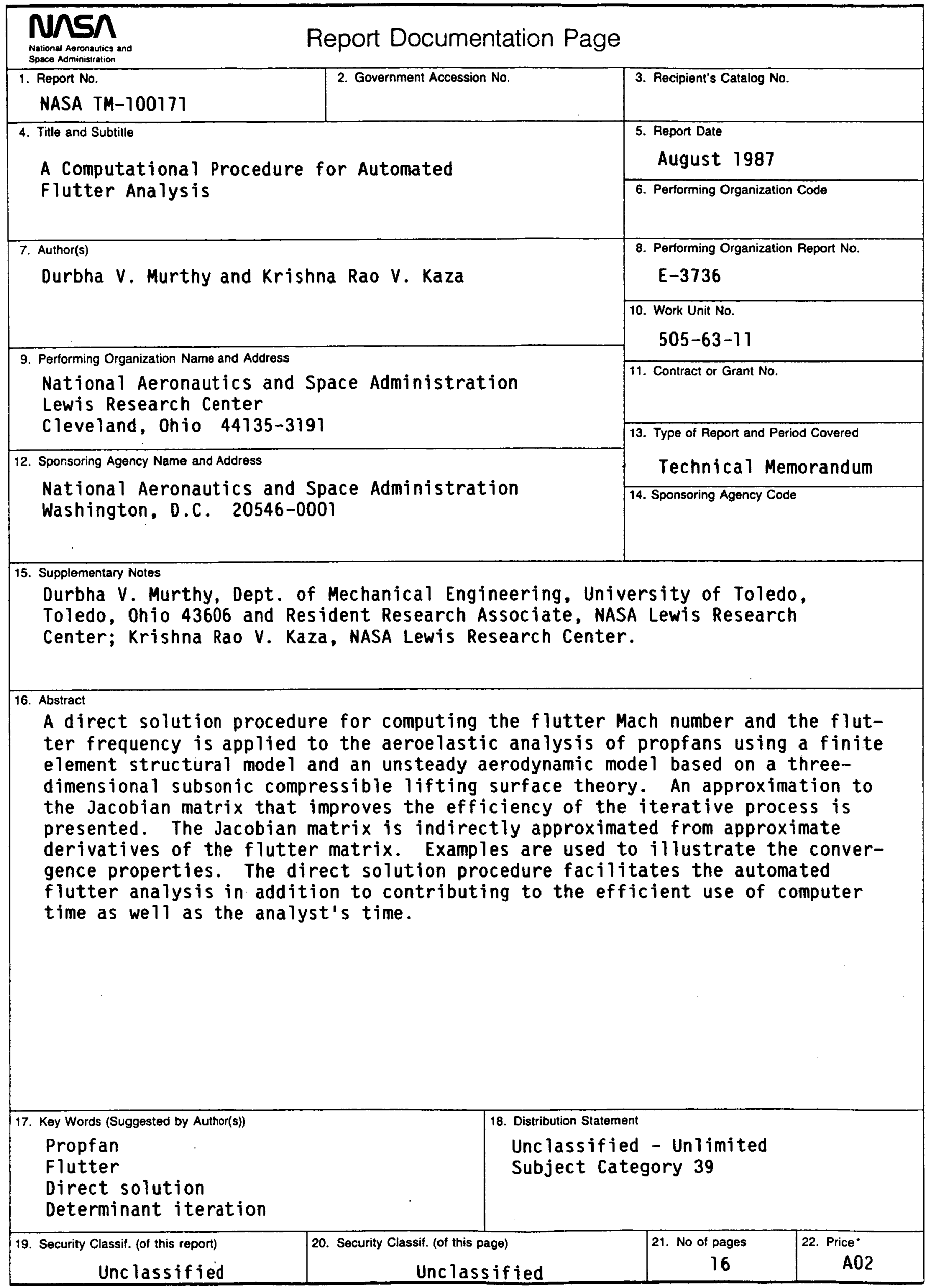

NASA FORM 1626 OCT 86 *For sale by the National Technical Information Service. Springfield, Virginia 22161 